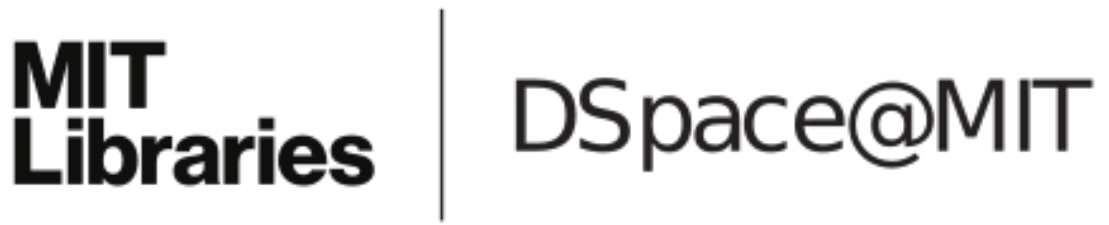

\author{
MIT Open Access Articles
}

Cortical circuits for the control of attention

The MIT Faculty has made this article openly available. Please share how this access benefits you. Your story matters.

Citation: Miller, Earl K, and Timothy J Buschman. "Cortical Circuits for the Control of Attention." Current Opinion in Neurobiology 23, no. 2 (April 2013): 216-222.

As Published: http://dx.doi.org/10.1016/j.conb.2012.11.011

Publisher: Elsevier

Persistent URL: http://hdl.handle.net/1721.1/102304

Version: Author's final manuscript: final author's manuscript post peer review, without publisher's formatting or copy editing

Terms of use: Creative Commons Attribution-Noncommercial-NoDerivatives 


\title{
Cortical Circuits for the Control of Attention
}

\author{
Earl K. Miller ${ }^{1}$ and Timothy J. Buschman ${ }^{2}$ \\ ${ }^{1}$ The Picower Institute for Learning and Memory and Department of Brain and Cognitive \\ Sciences, Massachusetts Institute of Technology, Cambridge, MA 02139 \\ ${ }^{2}$ Princeton Neuroscience Institute and Department of Psychology, Princeton University, \\ Princeton, NJ
}

\begin{abstract}
How are some thoughts favored over others? A wealth of data at the level of single neurons has yielded candidate brain areas and mechanisms for our best understood model: visual attention. Recent work has naturally evolved toward efforts at a more integrative, network, understanding. It suggests that focusing attention arises from interactions between widespread cortical and subcortical networks that may be regulated via their rhythmic synchronization.
\end{abstract}

\section{Introduction}

Everybody has experienced our brain's strict bottleneck that only allows a few simultaneous thoughts. An understanding of cortical function, then, ultimately depends on insight into how cortical networks coordinate to focus their processing on the same thought. The best understood model of this is visual attention.

Neurophysiological studies have provided a wealth of insight on the neural mechanisms of attention, correlates of which are seen in virtually all cortical areas that process visual information (i.e. most of cortex). This has led to increased experimental focus on how these widespread neurons form functional circuits. The consensus across a large number of studies, including both animal electrophysiology and human imaging work, suggests attention stems from a frontal-parietal control network acting on visual cortex. Within this network, synchronized oscillations (the "brain waves" long known to vary with attentional focus) may provide a substrate that allows interactions between specific regions, thereby selecting the neural representations that will win the competition for limited cognitive resources.

\section{Finding the top and bottom in attention networks}

Attention can be captured in a bottom-up fashion, by a salient stimulus. For example, brightly colored or fast moving objects are often important and are therefore salient. But intelligent behavior depends on top-down control signals that can modulate bottom-up sensory processing in favor of inputs more relevant to achieving long-term goals. Neurophysiological studies have begun to distinguish the circuitry, within a shared frontalparietal network, that guides top-down and bottom-up attention (Figure 1).

\footnotetext{
(C) 2012 Elsevier Ltd. All rights reserved.
}

Publisher's Disclaimer: This is a PDF file of an unedited manuscript that has been accepted for publication. As a service to our customers we are providing this early version of the manuscript. The manuscript will undergo copyediting, typesetting, and review of the resulting proof before it is published in its final citable form. Please note that during the production process errors may be discovered which could affect the content, and all legal disclaimers that apply to the journal pertain. 
Bottom-up attention signals may be first extracted in, and therefore flow from, the parietal cortex. One particular region, lateral intraparietal cortex (LIP), seems to contain saliency maps sensitive to strong sensory inputs [1] (Figure 2). Highly salient, briefly flashed, stimuli capture both behavior and the response of LIP neurons [2,3]. Microstimulating LIP biases visual search toward the corresponding location in the presumptive LIP saliency map [4]. Saliency maps are thought to automatically select the strongest sensory input via competition between map locations. This may result from interactions between excitatory receptive field centers (ERFCs) and inhibitory surrounds (Figure 2). The planning of a saccade to a location outside the ERFC suppresses LIP activity to a stimulus in the ECRF, reflecting LIP's center-surround structure [5]. Saliency maps are also seen in the frontal cortex $[6,7]$ as are center/surround interactions [8]. However, LIP neurons signal salient stimuli with a short latency $[9,10]$, shorter than the frontal cortex $[11,12]$. This suggests the neural signals reflecting the bottom-up capture of attention flows from parietal, not frontal, cortex. The saliency maps in parietal cortex may, in turn, be partially inherited from midbrain structures [13]: local inactivation of superior colliculus disrupts an animal's ability to select a salient stimulus [14].

By contrast, the network interactions for top-down shifts of attention networks seem to flow in a different direction: originating in frontal cortex, the brain region most associated with "executive" brain functions. Deactivation of the frontal eye fields (FEF) disrupts planned (top-down) saccades but has no effect on bottom-up stimulus detection [15]. Similarly, removing the top-down influence of the frontal cortex on visual cortex by combining unilateral PFC lesions with a split-brain transection results in monkeys that cannot flexibly switch their attention to constantly changing targets but has no effect when attention can be automatically grabbed by a salient, pop-out, target [16].

Neurophysiological studies also suggest that top-down signals may originate from the frontal cortex (Figure 1). Frontal cortical neurons reflect shifts of top-down attention with a shorter latency than parietal area LIP $[11,12]$. When attention is focused, the FEF and visual cortex go into rhythmic synchrony (more below) with a phase offset that suggests the former is driving the latter [17]. If internal control of attention originates in frontal cortex, artificial activation of frontal cortex should induce the type of top-down modulation of visual cortex seen during volitional shifts of attention. It does. Microstimulation of the FEF produces topdown attention-like modulation of visual area V4 [18]. This can also be seen by modulating dopamine in the FEF, the neurotransmitter system most associated with reward and goaldirected behavior [19].

But how exactly do top-down signals from frontal cortex influence visual cortex? First, it may act via a cascade down the visual cortical hierarchy instead of being "broadcast" simultaneously to multiple visual cortical areas. Just as volitional shifts of attention appear with a shorter latency in frontal cortex than in LIP [11], they are seen in LIP before visual cortical area MT [20]. Similar effects are seen in the ventral visual stream: attention to a visual stimulus first enhances the response of V4 neurons, then V2 neurons, and, finally, V1 neurons [21]. This cascade presumably allows the top-down signals to selectively enhance the level of stimulus representation (cortical hierarchy level) appropriate for the task at hand. Second, top-down signals seem to be excitatory in nature. Inputs from the FEF make excitatory synapses on pyramidal neurons in prefrontal, parietal and visual cortex, suggesting that top-down signals boost the activity corresponding to attended stimuli [22], perhaps after their amplification via local recurrent connections. Then, the suppression of unattended stimuli can occur via local lateral inhibitory interactions, perhaps via centersurround antagonism (see above; Figure 2). 


\section{Synchrony as a mechanism of attention}

It has long been known that brain waves (coordinated oscillations among many neurons) vary their frequency with cognitive focus. Recent studies have shown how the brain waves may reflect mechanisms that control network interactions.

Oscillations create synchronous spikes that can have a greater impact than unsynchronized spikes as they all arrive simultaneously at downstream neurons $[23,24]$. This coincident input is known to have a super-additive effect. Therefore, if sensory neurons tuned to the same stimulus synchronize their firing, that stimulus will be more strongly represented in downstream areas. In this fashion, local synchrony may help the brain to improve its signalto-noise ratio while, at the same time, reducing the number of spikes needed to represent a stimulus $[25,26]$. If true, then one might expect the spiking activity of synchronized neurons to carry more information than non-synchronized neurons. Indeed, this has been seen for remembered stimuli in the prefrontal cortex [27] and, in fact, only synchronous neurons in parietal cortex predict behavior in a reach-and-saccade task [28].

Synchronized spikes, then, seem ideal for focal attention because some stimulus representations must be enhanced at the expense of others. Support comes from observations that increased attention modulates oscillatory synchrony in visual cortex [29,30]. Neurons in area V4 with receptive fields encompassing an attended stimulus increased synchronized gamma band $(30-90 \mathrm{~Hz})$ oscillations [29]. By contrast, neurons representing an unattended stimulus showed increased low frequency $(<17 \mathrm{~Hz})$ synchronization. One explanation for this is that low frequency synchrony may reflect common and thus uninformative inputs to the neurons. Thus, the negative correlation between low frequency synchrony and attention may reflect a mechanism that improves information transmission by removing these common "noise" sources [31,32].

Different neural mechanisms may give rise to different frequency oscillations [33]. Higher frequency rhythms $(>30 \mathrm{~Hz})$ may reflect competitive interactions between inhibitory neurons [34], the same sort of competitive interactions that can capture salient stimuli (see above; Figure 2). In fact, high-frequency synchrony is seen within and between cortical and subcortical areas during bottom-up capture of attention $[11,29,35]$. In addition, high-frequency synchrony occurs within a cortical area between neurons that are sensitive to an attended stimulus [36]. What this all suggests is that higher frequency $(>30 \mathrm{~Hz})$ synchrony may result from the local interactions that underlie the computations of stimulus features within a cortical area. Top-down signals add energy to these circuits, boosting the synchrony of an attended object. Supporting this hypothesis, microstimulation of the frontal eye fields induces high-frequency oscillations in LIP when a target is in the neuron's receptive field [37].

\section{Coordinating network interactions via synchronized rhythms}

If attention engages networks throughout the cortex then it follows that there must be mechanisms that regulate network interactions, sculpting communication between brain areas so that top-down and bottom-up signals find their intended targets. Rhythmic synchrony may provide it. If two brain areas oscillate in phase they are more likely to influence one another and if they are out of phase, they are less likely to influence each other. This has led to the suggestion that inter-areal synchrony could be used to flexibly change the effective connectivity between areas [23,24]. Abnormalities in the oscillations could explain neuropsychiatric pathologies such as schizophrenia [38].

Support for this comes from observations that inter-areal oscillatory synchrony between prefrontal, parietal, and visual cortex increases with focal attention [11,20,39]. This 
synchrony can be quite specific, reinforcing communication between like and behaviorally relevant representations. For example, during visual attention, visual, not movement-related, neurons in the frontal cortex showed synchronized oscillations with the visual cortex. Furthermore, this synchrony is limited to neurons whose receptive fields overlap the attended object [40]. Synchrony between areas within visual cortex is also highly specific: sub-regions of V1 that represent an attended object will synchronize with downstream V4 neurons with overlapping receptive fields, even to the exclusion of other, competing but unattended, stimulus representations in V1 [41]. This specificity argues for a direct functional role for synchronized rhythms in attention.

But how is this coordinated across the cortex? Neuromodulators, such as acetylcholine, modulate cortical oscillations; synchrony may arise from it acting on several cortical areas simultaneously [42]. Inter-areal synchrony may also be regulated by subcortical structures, like the thalamus, that project widely and therefore are in an ideal position to regulate activity across cortical regions [43]. Recent support for this comes from observations that during visual attention there are increases in higher frequency synchrony between cortical areas along with an increase in low frequency synchrony between the thalamus and cortex. These low frequencies may provide a "carrier wave" on which higher frequency oscillations across cortex can become entrained [44]. Consistent with a thalamic generator, lowfrequency oscillations are stronger in the deeper layers of cortex that project to the thalamus [45]. However, as noted above, previous work has shown a decrease in low-frequency synchrony within visual cortex during sustained attention [29,36], necessitating future work to better understand the role of these oscillations.

In general, lower-frequency oscillations may reflect wider-ranging coordination than highfrequency oscillations. This is seen across regions, whether cortical-subcortical $[43,44]$ or cortical-cortical $[11,46]$. Low-frequency oscillations can also exploit rhythms in the external world, enhancing neural processing by putting neurons and stimuli in lockstep. When monkeys attend to rhythmic streams of either sequential visual or auditory stimuli, LFPs and spikes in cortex synchronize to the rhythm of the attended stream and not to the rhythm of an unattended stream [47]. Brain oscillations can also exploit, or even create, lockstep between the brain and behavior. During search of a visual display, shifts of covert ("mind's eye") attention and its correlate FEF neurons synchronize to lower-frequency, beta ( $25 \mathrm{~Hz})$ oscillations across the frontal cortex. This suggests a lockstep between neural activity and periodic sampling of the external world via an attentional spotlight [48]. Similar periodicity of attention has been observed in humans [49]. Indeed, much of sensory intake is periodic, whether regularly shifting attention and/or the eyes, touching a surface, or sniffing an odor. The correlation between neural oscillations and behavior suggests sampling the external world is periodic because the underlying neural processes are themselves rhythmic.

\section{Concluding Remarks}

We have discussed how visual attention may be focused via a frontoparietal network acting on visual cortex and how top-down and bottom-up attention may emanate from different parts of this network (frontal and parietal cortex, respectively). These network interactions may be regulated via rhythmic oscillations which may also support periodic sampling of the external world. This could extend to all cognitive processes, suggesting our brain does not operate continuously, but rather discretely, with pulses of activity routing packets of information [50]. Such discrete cycles would provide a backbone for coordinating computations (and their results) across disparate networks. This ensures that all needed information reaches the correct downstream neurons within the brief time window necessary for their integration (Figure 3). However, it comes at a cost: it's naturally limited in 
bandwidth; only so many things can be computed or carried in a single oscillatory cycle.

This can explain the most fundamental property of conscious thought, its limited capacity.

\section{References}

1. Arcizet F, Mirpour K, Bisley JW. A Pure Salience Response in Posterior Parietal Cortex. Cereb. Cortex. 2011; 21:2498-2506. [PubMed: 21422270]

2. Bisley JW, Goldberg ME. Neuronal Activity in the Lateral Intraparietal Area and Spatial Attention. Science. 2003; 299:81-86. [PubMed: 12511644]

3. Bisley JW, Goldberg ME. Neural Correlates of Attention and Distractibility in the Lateral Intraparietal Area. J Neurophysiol. 2006; 95:1696-1717. [PubMed: 16339000]

4**. Mirpour K, Ong WS, Bisley JW. Microstimulation of Posterior Parietal Cortex Biases the Selection of Eye Movement Goals During Search. J Neurophysiol. 2010; 104:3021-3028. [PubMed: 20861428] This work used microstimulation of LIP in monkeys performing a foraging task. Microstimulation made it more likely that monkeys would choose the stimulus in the LIP neuron's receptive field, as if that stimulus was salient.

5. Falkner AL, Krishna BS, Goldberg ME. Surround Suppression Sharpens the Priority Map in the Lateral Intraparietal Area. J. Neurosci. 2010; 30:12787-12797. [PubMed: 20861383]

6. Fecteau JH, Munoz DP. Salience, relevance, and firing: a priority map for target selection. Trends in Cognitive Sciences. 2006; 10:382-390. [PubMed: 16843702]

7. Thompson KG, Bichot NP, Schall JD. Dissociation of Visual Discrimination From Saccade Programming in Macaque Frontal Eye Field. J Neurophysiol. 1997; 77:1046-1050. [PubMed: 9065870]

8. Cavanaugh J, Joiner WM, Wurtz RH. Suppressive Surrounds of Receptive Fields In Monkey Frontal Eye Field. J. Neurosci. 2012; 32:12284-12293. [PubMed: 22933810]

9. Ipata AE, Gee AL, Goldberg ME, Bisley JW. Activity in the Lateral Intraparietal Area Predicts the Goal and Latency of Saccades in a Free-Viewing Visual Search Task. J. Neurosci. 2006; 26:36563661. [PubMed: 16597719]

10. Premereur E, Vanduffel W, Janssen P. Functional Heterogeneity of Macaque Lateral Intraparietal Neurons. J. Neurosci. 2011; 31:12307-12317. [PubMed: 21865473]

11. Buschman TJ, Miller EK. Top-Down Versus Bottom-Up Control of Attention in the Prefrontal and Posterior Parietal Cortices. Science. 2007; 315:1860-1862. [PubMed: 17395832]

12. Li L, Gratton C, Yao D, Knight RT. Role of frontal and parietal cortices in the control of bottomup and top-down attention in humans. Brain Research. 2010; 1344:173-184. [PubMed: 20470762]

13. Knudsen EI. Control from below: the role of a midbrain network in spatial attention. European Journal of Neuroscience. 2011; 33:1961-1972. [PubMed: 21645092]

14. McPeek RM, Keller EL. Deficits in saccade target selection after inactivation of superior colliculus. Nature Neuroscience. 2004; 7:757-763.

15. Lee K-M, Ahn K-H, Keller EL. Saccade Generation by the Frontal Eye Fields in Rhesus Monkeys Is Separable from Visual Detection and Bottom-Up Attention Shift. PLoS ONE. 2012; 7:e39886. [PubMed: 22761923]

16. Rossi AF, Bichot NP, Desimone R, Ungerleider LG. Top-Down Attentional Deficits in Macaques with Lesions of Lateral Prefrontal Cortex. J. Neurosci. 2007; 27:11306-11314. [PubMed: 17942725]

17. Gregoriou GG, Gotts SJ, Zhou H, Desimone R. High-Frequency, Long-Range Coupling Between Prefrontal and Visual Cortex During Attention. Science. 2009; 324:1207-1210. [PubMed: 19478185]

18. Moore T, Armstrong KM. Selective gating of visual signals by microstimulation of frontal cortex. Nature. 2003; 421:370-373. [PubMed: 12540901]

19. Noudoost B, Moore T. Control of visual cortical signals by prefrontal dopamine. Nature. 2011; 474:372-375. [PubMed: 21572439]

20. Saalmann YB, Pigarev IN, Vidyasagar TR. Neural Mechanisms of Visual Attention: How TopDown Feedback Highlights Relevant Locations. Science. 2007; 316:1612-1615. [PubMed: 17569863] 
21. Buffalo EA, Fries P, Landman R, Liang H, Desimone R. A backward progression of attentional effects in the ventral stream. PNAS. 2010; 107:361-365. [PubMed: 20007766]

22. Anderson JC, Kennedy H, Martin KAC. Pathways of Attention: Synaptic Relationships of Frontal Eye Field to V4, Lateral Intraparietal Cortex, and Area 46 in Macaque Monkey. The Journal of Neuroscience. 2011; 31:10872-10881. [PubMed: 21795539]

23. Fries P. A mechanism for cognitive dynamics: neuronal communication through neuronal coherence. Trends in Cognitive Sciences. 2005; 9:474-480. [PubMed: 16150631]

24. Salinas E, Sejnowski TJ. Impact of Correlated Synaptic Input on Output Firing Rate and Variability in Simple Neuronal Models. J. Neurosci. 2000; 20:6193-6209. [PubMed: 10934269]

25. Tiesinga PHE, Fellous J-M, Jos JV, Sejnowski TJ. Information transfer in entrained cortical neurons. Network: Computation in Neural Systems. 2002; 13:41-66.

26. Siegel M, König P. A Functional Gamma-Band Defined by Stimulus-Dependent Synchronization in Area 18 of Awake Behaving Cats. J. Neurosci. 2003; 23:4251-4260. [PubMed: 12764113]

27. Siegel M, Warden MR, Miller EK. Phase-dependent neuronal coding of objects in short-term memory. PNAS. 2009; 106:21341-21346. [PubMed: 19926847]

28**. Dean HL, Hagan MA, Pesaran B. Only Coherent Spiking in Posterior Parietal Cortex Coordinates Looking and Reaching. Neuron. 2012; 73:829-841. [PubMed: 22365554] Monkeys made combination eye and arm-reaching movements to a visual target. Only spikes synchronized to $15 \mathrm{~Hz}$ LFP oscillations were predictive of the animal's reaction time. This suggests that synchronized spikes carry more behaviorally relevant information than unsynchronized spikes.

29. Fries P, Reynolds JH, Rorie AE, Desimone R. Modulation of oscillatory neuronal synchronization by selective visual attention. Science. 2001; 291:1560. [PubMed: 11222864]

30. Womelsdorf T, Fries P, Mitra PP, Desimone R. Gamma-band synchronization in visual cortex predicts speed of change detection. Nature. 2006; 439:733-736. [PubMed: 16372022]

31. Cohen MR, Maunsell JHR. Attention improves performance primarily by reducing interneuronal correlations. Nature Neuroscience. 2009; 12:1594-1600.

32. Mitchell JF, Sundberg KA, Reynolds JH. Spatial Attention Decorrelates Intrinsic Activity Fluctuations in Macaque Area V4. Neuron. 2009; 63:879-888. [PubMed: 19778515]

33. Ainsworth M, Lee S, Cunningham MO, Traub RD, Kopell NJ, Whittington MA. Rates and Rhythms: A Synergistic View of Frequency and Temporal Coding in Neuronal Networks. Neuron. 2012; 75:572-583. [PubMed: 22920250]

34. Cardin JA, Carlen M, Meletis K, Knoblich U, Zhang F, Deisseroth K, Tsai L-H, Moore CI. Driving fast-spiking cells induces gamma rhythm and controls sensory responses. Nature. 2009; 459:663667. [PubMed: 19396156]

35**. Goddard CA, Sridharan D, Huguenard JR, Knudsen EI. Gamma Oscillations Are Generated Locally in an Attention-Related Midbrain Network. Neuron. 2012; 73:567-580. [PubMed: 22325207] Using brain slices that preserved circuits of the optic tectum in birds, the authors found that gamma band oscillations were generated locally. Oscillations start in the multisensory deeper layers and then project to superficial visual layers. They suggest that this opens a channel of communication with the cortex and other subcortical structures.

36. Bichot NP, Rossi AF, Desimone R. Parallel and Serial Neural Mechanisms for Visual Search in Macaque Area V4. Science. 2005; 308:529-534. [PubMed: 15845848]

$37 * * *$. Premereur E, Vanduffel W, Roelfsema PR, Janssen P. Frontal eye field microstimulation induces task-dependent gamma oscillations in the lateral intraparietal area. J Neurophysiol. 2012; 108:1392-1402. [PubMed: 22673327] Stimulation in the FEF induced gamma oscillations in area LIP but only when a saccade target was in the LIP receptive field. This suggests that top-down signals can amplify high-frequency oscillations in other cortical areas.

38. Uhlhaas PJ, Singer W. Abnormal neural oscillations and synchrony in schizophrenia. Nature Reviews Neuroscience. 2010; 11:100-113.

39. Siegel M, Donner TH, Oostenveld R, Fries P, Engel AK. Neuronal Synchronization along the Dorsal Visual Pathway Reflects the Focus of Spatial Attention. Neuron. 2008; 60:709-719. [PubMed: 19038226]

40***. Gregoriou GG, Gotts SJ, Desimone R. Cell-Type-Specific Synchronization of Neural Activity in FEF with V4 during Attention. Neuron. 2012; 73:581-594. [PubMed: 22325208] Monkeys 
focused their attention on a cued target. There was spike-LFP synchrony between FEF and area V4. When the authors separated neurons into those that had purely visual vs visuomotor or motor responses, it was found that only the pure visual neurons synchronized to area V4. The specificity of the synchrony for different cell types suggests a direct functional role for synchrony in top-down attention.

41***. Bosman CA, Schoffelen J-M, Brunet N, Oostenveld R, Bastos AM, Womelsdorf T, Rubehn B, Stieglitz T, De Weerd P, Fries P. Attentional Stimulus Selection through Selective Synchronization between Monkey Visual Areas. Neuron. 2012; 75:875-888. [PubMed: 22958827] Recordings were made between LFPs in areas V4 and V1. When monkeys attended to a particular stimulus at a particular location, there was oscillatory synchrony between the specific V4 and V1 recording sites that represented that location.

42. Whittington MA, Cunningham MO, LeBeau FEN, Racca C, Traub RD. Multiple origins of the cortical gamma rhythm. Developmental Neurobiology. 2011; 71:92-106. [PubMed: 21154913]

43. Saalmann YB, Kastner S. Cognitive and Perceptual Functions of the Visual Thalamus. Neuron. 2011; 71:209-223. [PubMed: 21791281]

44. Saalmann YB, Pinsk MA, Wang L, Li X, Kastner S. The Pulvinar Regulates Information Transmission Between Cortical Areas Based on Attention Demands. Science. 2012; 337:753-756. [PubMed: 22879517] Saalmann et al simultaneously recorded neural activity in a secondary thalamic region (the pulvinar) and two visual cortex areas (V4 and IT) while monkeys performed a discrimination task requiring attention. Attention was found to increase low-frequency synchrony between the thalamus and cortex. High-frequency synchrony between the two cortical regions was modulated by thalamo-cortical low-frequency synchrony. Therefore, low-frequency, interregional, synchrony may regulate locally generated higher-frequency oscillations.

45. Buffalo EA, Fries P, Landman R, Buschman TJ, Desimone R. Laminar differences in gamma and alpha coherence in the ventral stream. PNAS. 2011; 108:11262-11267. [PubMed: 21690410]

46. Pesaran B, Nelson MJ, Andersen RA. Free choice activates a decision circuit between frontal and parietal cortex. Nature. 2008; 453:406-409. [PubMed: 18418380]

47. Lakatos P, Karmos G, Mehta AD, Ulbert I, Schroeder CE. Entrainment of Neuronal Oscillations as a Mechanism of Attentional Selection. Science. 2008; 320:110-113. [PubMed: 18388295]

$48 * *$. Buschman TJ, Miller EK. Serial, Covert Shifts of Attention during Visual Search Are Reflected by the Frontal Eye Fields and Correlated with Population Oscillations. Neuron. 2009; 63:386396. [PubMed: 19679077] Monkeys were found to search a visual display by covertly shifting their attentional focus every $40 \mathrm{~ms}$. Neural correlates of this shift in FEF were synchronized to 25 $\mathrm{Hz}$ oscillations in local field potentials. This suggests that oscillations can provide a "clocking signal" that regulates or signals when attention is shifted.

49. Landau AN, Fries P. Attention Samples Stimuli Rhythmically. Current Biology. 2012; 22:10001004. [PubMed: 22633805]

50. Buschman TJ, Miller EK. Shifting the spotlight of attention: evidence for discrete computations in cognition. Front. Hum. Neurosci. 2010; 4:194. [PubMed: 21119775] 


\section{Highlights (for review)}

Visual attention may be focused via a frontoparietal network acting on visual cortex.

These network interactions may be regulated via rhythmic oscillations.

The brain may operate discretely with pulses of activity routing packets of information.

Oscillations are limited in bandwidth and may explain why conscious thought is limited in capacity. 


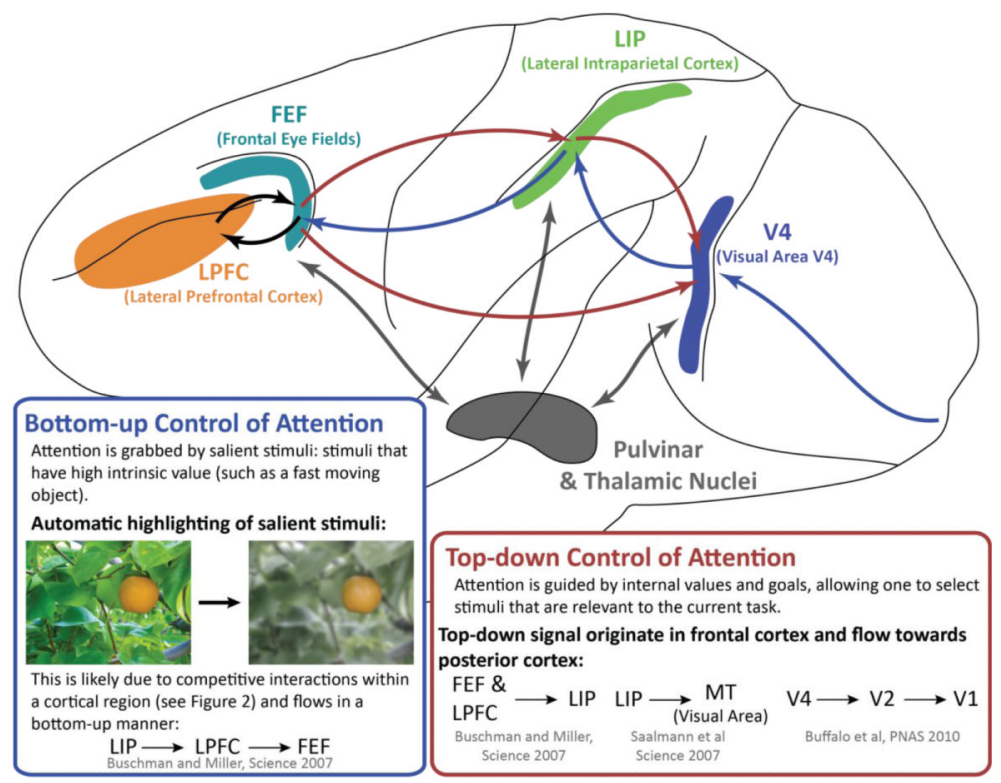

Figure 1.

Summary of interactions between brain regions that give rise to both bottom-up and topdown control of attention. 


\section{Computations in Local Circuitry and the Impact of Attention}

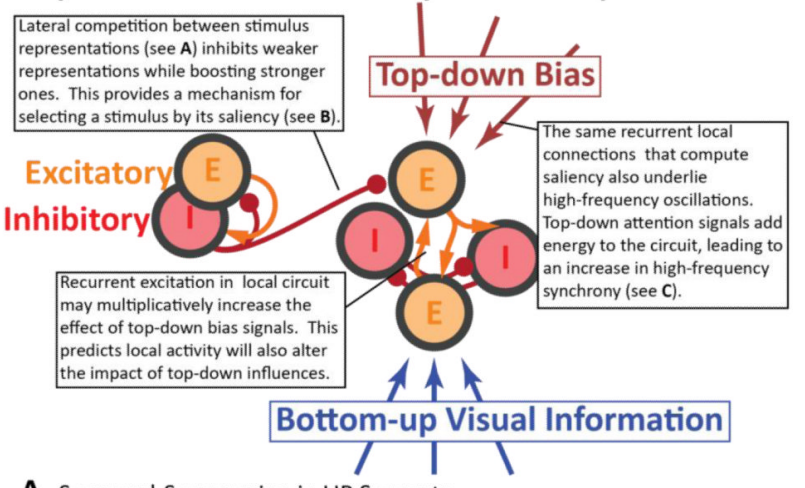

A Surround-Suppression in LIP Suggests Lateral Competition between Representations $\mathbf{C}_{\text {Electrical Stimulation of FEF }}$

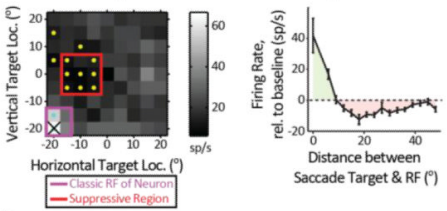

B LIP Neurons Encode Salient Objects

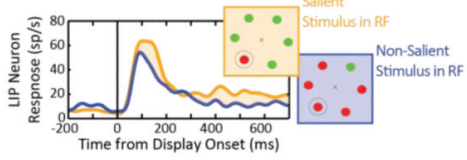
Induces High-frequency Oscillations in LIP

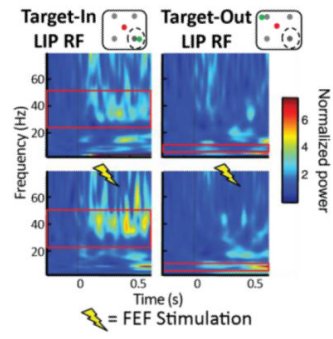

Figure 2.

Local, recurrent, circuitry within posterior cortex that gives rise to automatic selection of salient stimuli, possibly through rhythmic activity. (A) Center-surround structure of LIP receptive fields. (left) A single neuron that responds preferentially to stimuli briefly flashed in its receptive field (purple outline) and is inhibited by surrounding locations (red outline). This effect is consistent across the population (right). Adapted from Falkner et al, 2010 with permission. (B) LIP neurons encode salient objects, regardless of stimulus identity. Adapted from Arcizet et al, 2011 with permission. (C) Electrical stimulation of frontal eye fields induces high-frequency oscillations in parietal cortex. High-frequency oscillations are only increased when FEF is stimulated (bottom row) and the target is in the receptive field of the LIP recording site (left column). Adapted from Premeurer et al, 2012 with permission. 


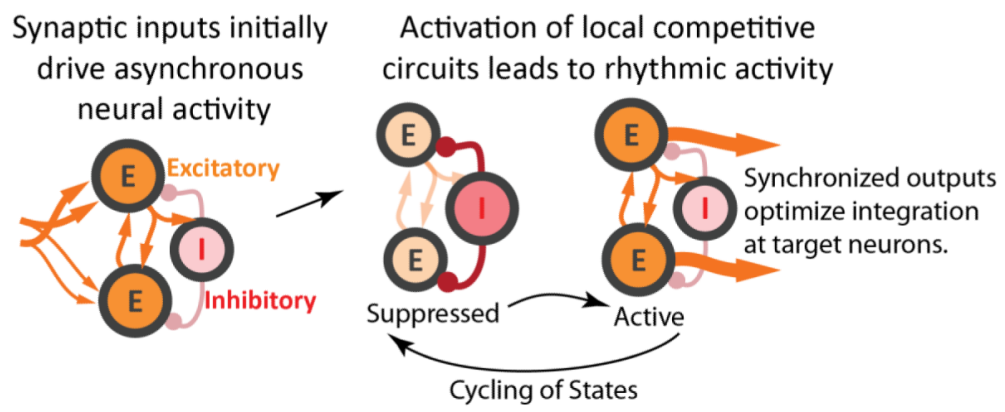

Figure 3.

Recurrent cortical circuitry leads to synchronous rhythms and discretization of neural processing. Initial inputs are asynchronous (left). By activating the local circuit this leads to alternating periods of suppression (middle) and activation (right), observed as rhythms in the local population. This process discretizes neural computations and ensures all information is simultaneously available for downstream neurons to act upon. Time flows to the right. Excitatory and inhibitory neurons are orange and red, respectively. Active neurons and connections are saturated colors; inactive neurons and connections are lighter and smaller. 